\title{
The road ahead
}

\section{Roberto Cingolani}

\section{In recent years, education in nanotechnology has evolved to reflect the new skill set required to perform multidisciplinary research. As the field further develops, it will need to evolve again.}

S cientific disciplines have for a long time been well separated. This distinction between physics, chemistry, biology, medicine and engineering is, however, an artefact created to order and classify apparently different phenomena and systems. Physics, chemistry and biology explain most of the interactions and processes that rule the formation of complex systems at the microscopic level, and in turn, provide the knowledge base for medicine and engineering that describe macroscopic properties of both living and non-living systems. In the past few decades, these traditionally distinct research fields have begun to meet on a common ground - the observation, modelling and manipulation of matter at the nanoscale.

The reasons for this convergence are manifold, and a comprehensive discussion of them is beyond the scope of this Commentary. In short, scanning probe methods have provided the ability to observe atoms, molecules and their interactions on the laboratory bench top. Supercomputers have become ever more powerful and accessible, making simulation and modelling of nanoscale phenomena precise and reliable. Nanofabrication tools have been refined to sub-10 $\mathrm{nm}$ resolution. Advances in molecular biology have pushed life sciences towards quantum mechanics. The field of colloidal chemistry has developed a large variety of nanoparticles with atomically controlled size, shape and composition, which allows the structural, chemical and physical properties of matter to be tailored. As a result, scientists from different fields can now use similar tools to study a material regardless of its chemical or physical nature, be it a semiconductor nanostructure, a synthetic molecule or an enzyme. This cross-fertilization has produced a creative disorder that is driving science in new and unexpected directions.

Education could not be expected to follow such a revolution at the same pace. Initially, in the 1990s, undergraduate programmes maintained traditional specialized courses, and only at postgraduate level was there an attempt to foster interdisciplinary training. Lately, nanotechnology courses have been blossoming all over the world, trying to give a wider and interdisciplinary organization to university curricula. Today web searches can easily find hundreds of courses (bachelors, masters and PhDs) in nanotechnology - around 100 in the US, 100 in Europe, 60 in Asia and more than 20 in South America.

\section{From disorder to order}

Even though today different research fields share methods, tools and concepts to produce new ideas and to create new technologies, it is likely that in the next few years a degree of order will emerge from the current creative disorder (Fig. 1). The ever increasing awareness of the potential of nanotechnology in several fields and applications will lead to the development of university education and research training into more focused nanotech domains - nanomedicine or nanomaterials, for example. Something similar happened in engineering research over the past century: after an explosive period of development, which led to the realization of, for example, internal combustion engines, transistors and plastics, the field reorganized into many distinguishable domains such as mechanical, electrical and chemical engineering. Indeed, many of the nanotechnology orientated degrees already available focus on a specific domain of application.

To envisage how training and education should be organized today, we need to predict the challenges of tomorrow. One of the ultimate goals of nanoscience and nanotechnology is global sustainability. In particular, we must optimize our use of natural resources, such as water, food and natural fibres, and we must transform waste for useful means. This requires the fields of catalysis, chemistry and materials science to create biodegradable materials,

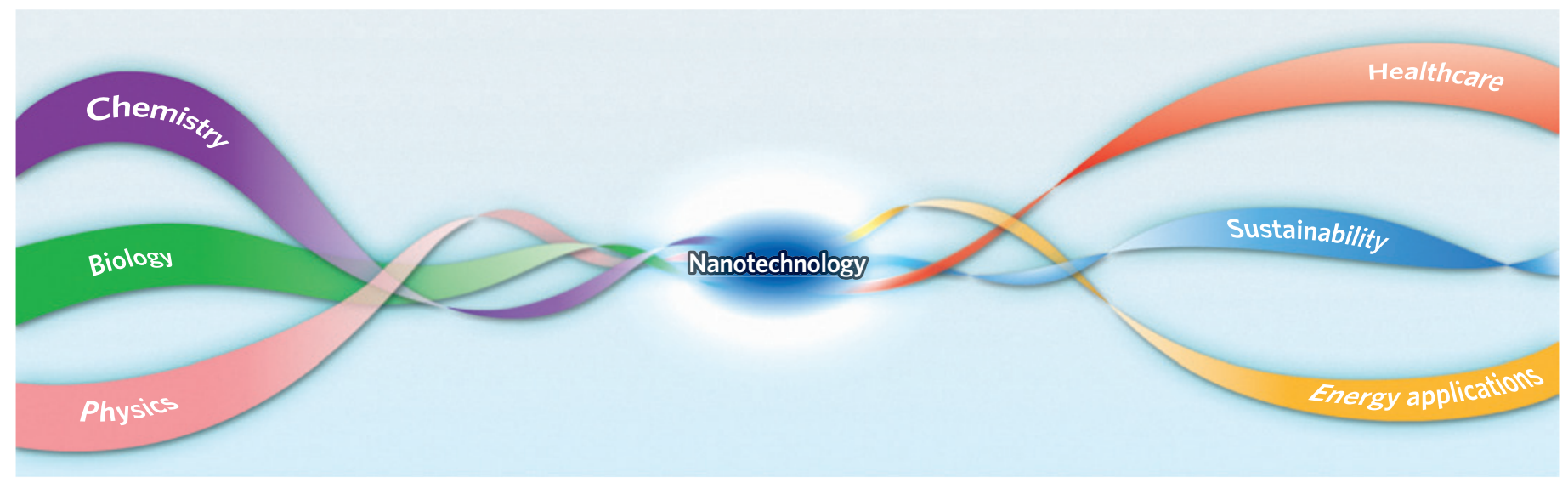

Figure 1 | The evolution of academic education in nanotechnology. Recently, traditionally distinct disciplines have met on a common ground - the manipulation of matter at the nanoscale. In the future, education in nanotechnology may be organized in courses that focus on specific challenges. 
to remove pollutants from water, and to produce and store energy. Energy applications must not focus solely on automobiles, but should extend to portable energy sources for powering sub-kW systems (such as domestic appliances), which represent a remarkable portion of the overall power consumption in advanced countries. Batteries and fuel cells that use bacteria or organic materials, and energy harvesting based on flexoelectric materials and thermoelectric devices should become technology of high priority. Energy conservation will require new insulating materials, self-cleaning surfaces, and more integrated solutions aimed at minimizing power dissipation and heat absorption together with ultralight composite materials with tailored mechanical properties to reduce fuel consumption in transport. Nanotechnology has a role to play in all of this.

Furthermore, nanotechnology for health and welfare will become a priority with the increasing life expectancy of humans. Strategies will be needed to guarantee sustainable and comfortable ageing of our populations, including early-stage diagnostics as well as personalized molecular therapies to fight neurodegeneration and cancer.

Even such a short compilation of priorities suggests that a conventional education might be insufficient for future nanotechnologists. The challenge is to create cross-disciplinary background knowledge for the next generation, so that they can face problems in a completely new and open way.

Physics, chemistry, mathematics and biology should be taught to all future scientists, although with different depth depending on the mathematical background required by the various disciplines. Life scientists will need to have an understanding of quantum mechanics so that they can exploit the power of nanotechnology in designing new biomedical applications. Similarly, engineers and materials scientists will need a wide knowledge of life science to profitably apply nanotechnology to living systems and to create bioinspired technologies. Basic tools for programming and computation should complete the education of future students.

Such broad training should not be considered dangerous or dispersive: scientists in the future will need a comprehensive vision of worldwide problems and common tools of investigation to address the problems in a multidisciplinary, parallel fashion.

\section{Challenges}

It is likely that all this training would be too much for a standard undergraduate curriculum. Education and training at this level should, therefore, rely on a long-term educational strategy involving the entire pipeline of the undergraduate degree and $\mathrm{PhD}$; a scientist that has a deep knowledge of nature and broad cross-disciplinary education cannot be educated in only $4-5$ years. Finding a reasonable balance between specialization and interdisciplinary education will be mandatory; standard classes might not be sufficient, and personalized training could be necessary depending on whether students will continue in research or will enter the industrial job market.

Finally, the large differences in the educational systems in the US and Europe also need to be taken into account. For example, the current US system, where, in general, science students have a wider science background (slightly less specialized at the beginning) compared with European students, may already be closer to the ideas presented above. However, the increasing interdisciplinary trend of research will have an impact on both educational systems, and change will probably be required soon.

Whether educational institutions and the job market will acknowledge and accept this transformation will be clear in a few years. For those who work in research and education, the challenge now is to educate new generations of scientists who are equipped with open minds and an interdisciplinary approach such that they are capable of making the most of all the possibilities that nanotechnology has to offer.

Roberto Cingolani is at the Italian Institute of Technology (IIT), Via Morego, 3016163 Genova, Italy.

e-mail: roberto.cingolani@iit.it 